\title{
CONVEX COMPONENTS, EXTREME POINTS, AND THE CONVEX KERNEL
}

\author{
JOHN W. KENELLY, W. R. HARE, JR., B. D. EVANS, \\ AND W. H. LUDESCHER ${ }^{1}$
}

In this paper, it is proved that the convex kernel of a compact $d$-dimensional starshaped set is the set of points that see all the $(d-2)$-extreme points. Certain maximal convex subsets are classified as being intersectionally independent, and the number of these present in a starshaped set leads to an upper bound on the dimension of the convex kernel.

Any starshaped set is related to a particular convex set, i.e., its convex kernel. With a review of the basic definitions given below, we see that this association suggests two general problems. First is the selection of a convex set and the analysis of whether it is the convex kernel of some starshaped set. The second is selection of a starshaped set and the analysis of its convex kernel; in particular the dimension characteristics or the construction of this related convex set. General results related to the first problem are given by Klee [1] and Post [2]. Particular conditions related to the dimension question posed in the second problem are given by Foland-Marr [3], Hare-Kenelly [4] and Larman [5]. Generalizations of these conditions are given by Toranzos [6]. In this paper both facets of the second problem are studied. The first section is directed toward the following consideration. It is known that the convex hull of a compact set is constructible by taking the convex hull of its extreme points. Realizing that the convex kernel is the intersection of the stars of all the points of the set, the question is posed whether the convex kernel is the intersection of the stars of the extreme points. An affirmative answer is given for $k$-extreme points and a negative answer is presented for extreme points. The second section recalls that the convex kernel is the intersection of all the convex components and with this fact gives a result that relates convex components to the dimension problem.

Presented in part to the Society, November 18, 1967 under the title Convex kernels and extreme points, and January 23, 1968 under the title $A n$ upper bound on the dimension of the convex kernel; received by the editors January $28,1968$.

1 The initial investigation of these results took place during an undergraduate research participation project at Clemson University, summer 1967. This work was supported in part by NSF Grant GY 3016. Mr. Evans was a student participant from Oklahoma State University and Mr. Ludescher was a student participant from Loras College. 
In a linear space, we say that $x$ sees $y$ via $S$ whenever $x$ and $y$ are points of $S$ and the segment $x y$ is contained in $S$. The totality of points of $S$ that $x$ sees via $S$ is taken to be the $S$-star of $x$, i.e., $S(x)$ $=\{y: x y \subset S\}$. The set $S$ is said to be starshaped whenever there is some point $x$ that sees all of $S$, i.e., $S(x)=S$. In this case the collection of all such points is termed the convex kernel, $\operatorname{ck}(S)=\{x: S(x)=S\}$. A convex component is taken to be a maximal convex subset of $S$. With this it can be shown that the convex kernel is convex, and may be characterized as either the intersection of the stars of all the points of $S$, or the intersection of all the convex components of $S$. See Valentine [7] and Toranzos [8].

1. Convex kernel and extreme points. The following definition includes modifications of previous definitions given by Asplund [9] and Fan [10]. It should be noted that the definition applies to nonconvex sets, so 0 -extreme and extreme points are not identical.

Definition 1.1. A point $p$ in a compact set $S$ is called a $k$-extreme point if and only if it is not the centroid of some nondegenerate $(k+1)$-simplex in $S$, i.e., a 0 -extreme point is not interior to a segment in $S$. The point $p$ is an extreme point if and only if $x \in S, y \in S$, $p=\beta x+(1-\beta) y \in S$ and $0<\beta<1$ imply $x=y=p$.

THEOREM 1.2. If $S$ is a compact starshaped set in d-dimensional Euclidean space, $R^{d}(d \geqq 2)$, then $\operatorname{ck}(S)=\bigcap_{\alpha \in A} S\left(e_{\alpha}\right)$ where $\left\{e_{\alpha}: \alpha \in A\right\}$ is the set of $(d-2)$-extreme points of $S$.

Proof. Since $\operatorname{ck}(S)=\bigcap_{x \in S} S(x)$, we need only show that $E \equiv \bigcap_{\alpha \in A} S\left(e_{\alpha}\right) \subset \operatorname{ck}(S)$. Take $w \in E \sim \operatorname{ck}(S)$. Since $w \notin \operatorname{ck}(S)$, there exists $y \in S$ such that $w y \nsubseteq S$. By the compactness of $S$, we take $y$ to be such that $\beta w+(1-\beta) y \notin S$ for arbitrarily small $\beta>0$. Take $k \in \operatorname{ck}(S) \neq \varnothing$, and consider the ray $k y=\{(1-\beta) k+\beta y: \beta \geqq 0\}$. Since $k \in \operatorname{ck}(S)$, we have $k y \cap S=k q$, a closed segment. For the first case consider $q$ to be different from $y$. The point $q$ cannot be a $(d-2)$ extreme point, for this would give $w q \subset S$. This results in $k$ seeing the segment $w q$. This implies that $w y \subset S$, contradicting wy $₫ S$. Thus $q$ is the centroid of a $(d-1)$-simplex $\sigma$ which is a subset of $S$. Letting $P$ denote the plane through $w, q$, and $k$, it follows that $P \cap \sigma$ contains an open segment relint $a b$ such that $a b \nsubseteq F(k, q)$. Here relint indicates the relative interior with respect to the smallest containing flat and $F(k, q)$ is the line established by $k$ and $q$. But this implies that for sufficiently small $\beta_{0}>0, \beta w+(1-\beta) y \in S$ for every $\beta$ satisfying $0<\beta<\beta_{0}$, contradicting the choice of $y$. Thus we conclude that $q=y$.

Since wy $₫ S$, we see that $y$ is not a $(d-2)$-extreme point. Now consider the nondegenerate $(d-1)$-simplex that is contained in $S$ 
with $y$ as its centroid. This simplex establishes a hyperplane $H$ and as in the previous case, we examine the intersection of the wky-plane, $P$, and the hyperplane $H$. The choice of $y$ insures that $y$ is a boundary point of $P \cap S$. We observe that $H \cap P$ is a line, and from this conclude that $H$ separates $w$ and $k$. For $y$ is interior to a segment contained in $S \cap P$, and nonseparation above would, as before, give points of $w y \cap S$ arbitrarily close to $y$. Now construct the following regions. First, $W^{-}$is taken to be a closed half-space opposite from $w$ with respect to a hyperplane containing $k y$ and not $w$. Then, $K^{-}$is taken to be a closed half-space opposite from $k$ with respect to a hyperplane containing wy and not $k$. The set $T \equiv K^{-} \cap W^{-} \cap S \cap P$ is a nonempty compact set. The generalized Krein-Milman theorem [10, p. 146] states that $T$ has an extreme point, $t$. Since any extreme point of $T$ is obviously a $(d-2)$-extreme point of $S$, we have $w t \subset S$. Thus $k$ sees $w t$ and $w y \subset S$.

All the hypotheses of the theorem seem to be necessary. A closed half-space shows that compactness is needed to insure the existence of any type of extreme points. The planar set

$$
\begin{aligned}
B & \equiv\{(x, y): x+y \leqq 3, x \geqq 0, y \geqq 0\} \\
& \sim\{(x, y): x+2 y<3, x>1, y>0\}
\end{aligned}
$$

shows that starshapedness is required. That is $B$ is a nonstarshaped set, but the stars of its 0 -extreme points intersect in a nonempty set. The set $C \equiv B \sim\{(x, y): 1<x<3, y=0\}$ illustrates that extreme points are not sufficient. A complicated three space example is needed to show that 0 -extreme points fail to allow a construction of the convex kernel. First construct a tetrahedron, i.e., $D \equiv$ convex hull $\{(24,0,0),(0,24,0),(0,0,24),(0,0,0)\}$. Remove the plane interior of $\operatorname{conv}\{(24,0,0),(0,24,0),(0,0,0)\}$. Here conv represents the convex hull. Then remove the interior of $\operatorname{conv}\{(24,0,0),(0,0,0)$, $(0,24,0),(16,4,1)\} \cup \operatorname{conv}\{(0,24,0),(0,0,0),(8,8,4),(16,4,1)\}$. The remaining set has four 0 -extreme points, i.e., the vertices of the original tetrahedron. These four points can see $(0,12,6)$, but this is not a kernel point since it fails to see $(16,4,1)$. This type of example seems to generalize, but it is already unduly complicated. Thus no proof is given that in general $(d-2)$-extreme points are needed.

2. Convex kernel and convex components. This section relates convex components to the dimension of the convex kernel. The earlier papers state finite point conditions related to the dimension of the convex kernel, and the most general result is due to Toranzos [6]. The main theorem of this section is stated in terms of convex 
components, but we demonstrate that the theorem generalizes the point condition results.

The results are given in finite dimensional linear spaces. Flats are translates of subspaces, and the linear dimension of the related subspace is assigned to the flat. The empty set has dimension -1 . A set $S$ is associated with its smallest containing flat, $F(S)$, and the corresponding dimension is assigned to the set, i.e., $\operatorname{dim}(S)=\operatorname{dim}(F(S))$. A collection of intersecting flats is said to be intersectionally independent, (i.i.), when no one of the flats contains the intersection of the remaining flats. A single flat is taken to be an intersectionally independent collection. A collection of sets is called intersectionally independent provided the collection of containing flats is intersectionally independent. We see that intersectional independence is preserved when we take subcollections. Likewise intersectional independence is preserved when we replace some of the sets with the intersection of their containing flats, i.e., $\left\{S_{1}, \cdots, S_{k}\right\}$ (i.i.) gives $\left\{\bigcap_{i=1}^{j} F\left(S_{i}\right), S_{j+1}, \cdots, S_{k}\right\}$ (i.i.)

LеммA 2.1. If $\left\{S_{i}\right\} 1 \leqq i \leqq k$ is an intersectionally independent collection of sets, then

$$
\operatorname{dim}\left(\bigcap_{i=1}^{k} F\left(S_{i}\right)\right) \leqq \min _{i}\left(\operatorname{dim}\left(S_{i}\right)\right)-k+1 .
$$

Proof. In case $k=1$, the result is trivial. So we assume without loss of generality that $\min _{i}\left(\operatorname{dim}\left(S_{i}\right)\right)=\operatorname{dim}\left(S_{1}\right)=s$. Observe that $\operatorname{dim}\left(F\left(S_{1}\right) \cap F\left(S_{2}\right)\right) \leqq s-1$. For otherwise, we would have a flat properly containing a subflat of the same dimension. Since $\left\{F\left(S_{1}\right) \cap F\left(S_{2}\right)\right.$, $\left.F\left(S_{3}\right)\right\}$ is an intersectionally independent collection, we have $\operatorname{dim}\left(\left(F\left(S_{1}\right) \cap F\left(S_{2}\right)\right) \cap F\left(S_{3}\right)\right) \leqq \operatorname{dim}\left(F\left(S_{1}\right) \cap F\left(S_{2}\right)\right)-1 \leqq s-2$. A simple induction formally completes the proof.

Recall that the convex kernel is the intersection of all the convex components, and is empty for nonstarshaped sets. Thus the convex kernel is contained in the intersection of any collection of convex components. Likewise its containing flat is contained in the intersection of the collection of flats that contain the components. This results in the following theorem.

ThEOREM 2.2. In a finite dimensional linear space; whenever a set $S$ contains $k$ intersectionally independent convex components $\left\{S_{i}\right\}$, $1 \leqq i \leqq k$, we have $\operatorname{dim}(\operatorname{ck}(S)) \leqq \min _{i}\left(\operatorname{dim}\left(S_{i}\right)\right)-k+1$.

For a starshaped set $M$ in a linear space, Toranzos [6] introduces property $\alpha_{k}$ as the following statement. For each affinely independent $k+2$ points $\left\{x_{1}, x_{2}, \cdots, x_{k+2}\right\} \subset M, \operatorname{dim} \bigcap_{i=1}^{k+2} S\left(x_{i}\right) \leqq k-1$. This 
property gives an upper bound on the dimension of the convex kernel. We state Toranzos's theorem as the following.

CoROllaRy 2.3. If $M$ is a starshaped set with property $\alpha_{k}$ and $\operatorname{dim}(M)>k$, then $\operatorname{dim}(\operatorname{ck}(M)) \leqq k-1$.

Proof. Since there is at least one convex component $K$ in $M$, property $\alpha_{k}$ readily gives $\operatorname{dim} K \leqq k$. Thus we have $\operatorname{dim}(\operatorname{ck}(M)) \leqq k-1+1$ $=k$. The set $M$ cannot be a convex set, so we must have two distinct convex components $K_{1}$ and $K_{2}$. If the components are intersectionally independent, then the $\operatorname{dim}(\operatorname{ck}(M)) \leqq k-1$. So assume that the components are not intersectionally independent. The components must contain the nonempty kernel, so this assumption gives (with adjustments on subscripts if necessary) $F\left(K_{1}\right) \subset F\left(K_{2}\right)$. If the containment is proper we have $\operatorname{dim}\left(K_{1}\right) \leqq k-1$ and the resulting bound $\operatorname{dim}(\operatorname{ck}(M))$ $\leqq(k-1)-1+1=k-1$. Equality, i.e., $F\left(K_{1}\right)=F\left(K_{2}\right)$, for all components $K$ gives $\operatorname{dim} M=k$, and the hypothesis is violated.

Examples can be readily constructed to show that the bound established in Theorem 2.2 is the exact dimension of the convex kernel for particular sets and for other sets the bound is in excess of the actual dimension of the convex kernel. It should also be noted that there exist planar compact starshaped sets with the following properties. Any finite number of convex components intersect in a set that has dimension greater than the kernel. Any finite number of extreme points see jointly a set that has a dimension greater than the kernel.

\section{REFERENCES}

1. V. L. Klee, $A$ theorem on convex kernels, Mathematika 12 (1965), 89-93.

2. K. A. Post, Star extension of plane convex sets, Nederl. Akad. Wetensch. Indag. Math. 26 (1964), 330-338.

3. N. E. Foland and J. M. Marr, Sets with zero dimensional kernels, Pacific J. Math. 19 (1966), 429-432.

4. W. R. Hare, Jr. and J. W. Kenelly, Concerning sets with one point kernel, Nieuw Arch. Wisk. 14 (1966), 103-105.

5. D. G. Larman, On the convex kernel of a compact set, Proc. Cambridge Philos. Soc. 63 (1967), 311-314.

6. F. A. Toranzos, The dimension of the kernel of a starshaped set, Notices Amer. Math. Soc. 14 (1967), 832.

7. F. A. Valentine, Convex sets, McGraw-Hill, New York, 1964.

8. F. A. Toranzos, Radial functions of convex and starshaped bodies, Amer. Math. Monthly 74 (1967), 278-280.

9. E. Asplund, $A$ k-extreme point is the limit of $k$-exposed points, Israel J. Math. 1 (1963), 161-162.

10. K. Fan, Convex sets and their applications, Argonne Natl. Lab. Appl. Math. Div. Summer Lectures, 1959.

Louisiana State University, New Orleans aNd Clemson University 